\title{
Technical troubles encountered in the seismic observation network of Pacific21
}

\author{
Yoshiki Yuki $^{1 *}$, Yasushi Ishihara ${ }^{1}$
}

\begin{abstract}
Real-time monitoring of broadband seismographs through the Internet enables us to discover various technical troubles of broadband seismic observation system of Pacific21 and to take measures to solve them at the next field maintenance occasions. This paper summarizes the characteristics and the countermeasures of four typical troubles that we discovered from real-time monitoring or encountered in our maintenance work. One trouble was a deviation of seismometer's response owing to insufficient insulation of its connector. It was very effective to protect the connectors from moisture as a preventive measure. Another trouble was that spike-like noise was generated in the seismometer's output when some equipment demanded high current from the power supply. In order to remove the noises, we have two solutions: to disconnect extra impedance from the grounding line for power return, and to improve the power supply. A third was high frequency noise from one-sided connection of differential signaling. It is important to check the waveforms diligently to find this trouble as soon as possible. The other was that the data logger's dedicated hard disk often stopped for a maximum of several hours. The frequency of hard disk's shutdown seems to depend on its firmware version. For this trouble, we learned that we should operate equipment with various firmware versions for some time before installation.
\end{abstract}

Keywords : seismometer, noise, differential signaling, data gap, Pacific21

Received 18 February 2009 ; Accepted 27 August 2009

1 Institute for Research on Earth Evolution, Japan Agency for Marine-Earth Science and Technology

Corresponding author :

Yoshiki Yuki

Institute for Research on Earth Evolution, Japan Agency for Marine-Earth Science and Technology

3173-25 Showa-machi, Kanazawa-ku, Yokohama 236-0001, Japan

$+81-45-778-5390$

yukiy@jamstec.go.jp

Copyright by Japan Agency for Marine-Earth Science and Technology 


\section{Introduction}

Pacific21 is a geophysical observation network which consists of broadband seismic stations, electromagnetic stations, and GPS stations in the Western Pacific region. Our team, the Earth Informatics and Geophysical Network Research Team, has been operating the Pacific21 network and providing the data of 26 seismic, 7 electromagnetic, and 8 GPS stations to the public on our Web site (http:// www.jamstec.go.jp/pacific21) (Mizutani et al., 2007). As of August 2009, we have been maintaining 14 seismic stations and 4 electromagnetic stations. Our seismograph system of these seismic stations consists of three parts of equipment: (1) Streckeisen's STS-1, STS-2, or Guralp CMG3T seismometers, (2) Kinemetrics/Quanterra Q330 data loggers that have 24-bit analog-to-digital (AD) converters, or the Q330HR that has 26-bit AD converters, and (3) the AC-to-DC (AC-DC) converter power controllers that have uninterruptible function or solar power generation (Fig. 1). In addition, we store data in the Q330's or Q330's dedicated hard disk (PB-14) as well to transmit data to our institute via the Internet from almost every station (Ishihara et al., 2005).

Until about five years ago when we began utilizing Internet technology for our telemetry, all data from stations was available for us either by asking collaborators at the stations to send us a data disk or by visiting the stations directly. Without real-time monitoring, it was sometimes too late to find the troubles which had disturbed seismic observations, and then it took quite some time until we could solve the trouble and restart observations. After connecting
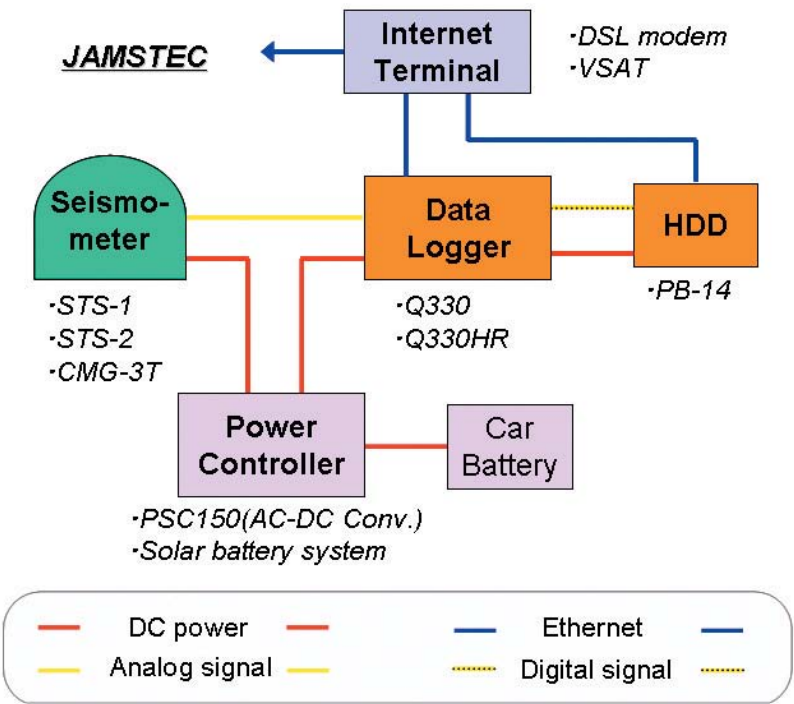

Fig.1. Diagram of the equipment at 14 broadband seismic stations that we have maintained. the stations via the Internet, we have been receiving data from the stations with a delay of only a few seconds. Owing to this, we can identify many kinds of troubles of stations immediately and prepare the measures to solve them properly before next maintenances of stations.

With this background, it is necessary to share our knowledge of various troubles which occurred at stations and possible measures to solve them with the members in our group, so as to help us to specify various troubles properly and to solve them more easily. Therefore, this paper describes several troubles which we had encountered during approximately the past five years. In this paper, we define a trouble not as an equipment breakdown that requires replacement with a new one, such as damage to equipment because of lightning, but as anomalous behavior of equipment, unusual noise generation in seismograms, and data gaps in our system originating from various sources.

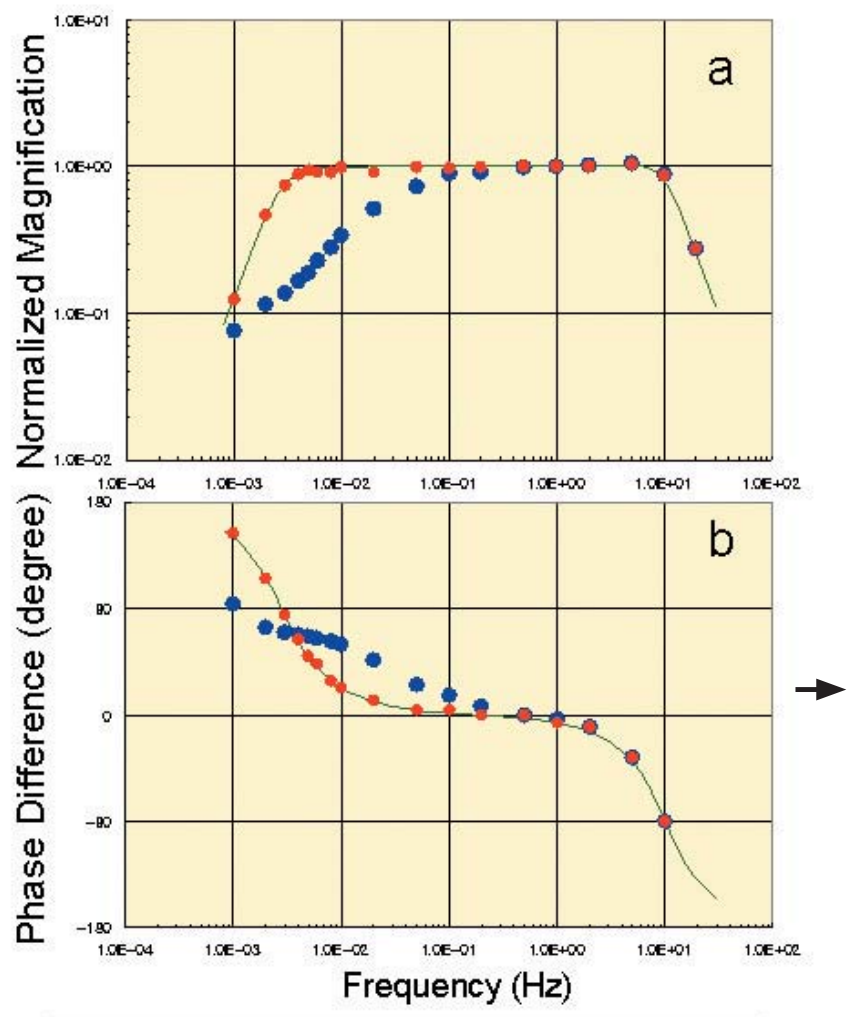

\section{Before replacement After replacement Theoretical response}

Fig.2. Frequency responses of STS-1 seismometer before and after replacing its base plate with connector. (a) Frequency response in amplitude. The value of the vertical axis equals amplitude magnification of output signal to input signal normalizing by that in frequency of $1 \mathrm{~Hz}$. (b) Frequency response in phase. 


\section{Troubles at broadband seismic stations}

\subsection{Deviation of seismometer's frequency response due} to high humidity

Our seismograph, specifically the STS-1 seismometer, has frequently suffered from the troubles of moisture since all seismic vaults of Pacific2 1 have very high humidity where some parts of equipment, including seismometers, are installed. From our maintenance work we found that this high humidity could result in poor insulation between some conductors in the STS-1 seismometer's multi-pin connector. In many cases, we improved many connectors with insufficient insulation by drying them with desiccant such as silica gel, based on our experiments (Yuki and Ishihara, 2005). However, we were not able to improve some of the connectors on the STS-1 seismometer's base plate simply by drying them. Finally we had to replace the base plate since that connector cannot be removed. Both before and
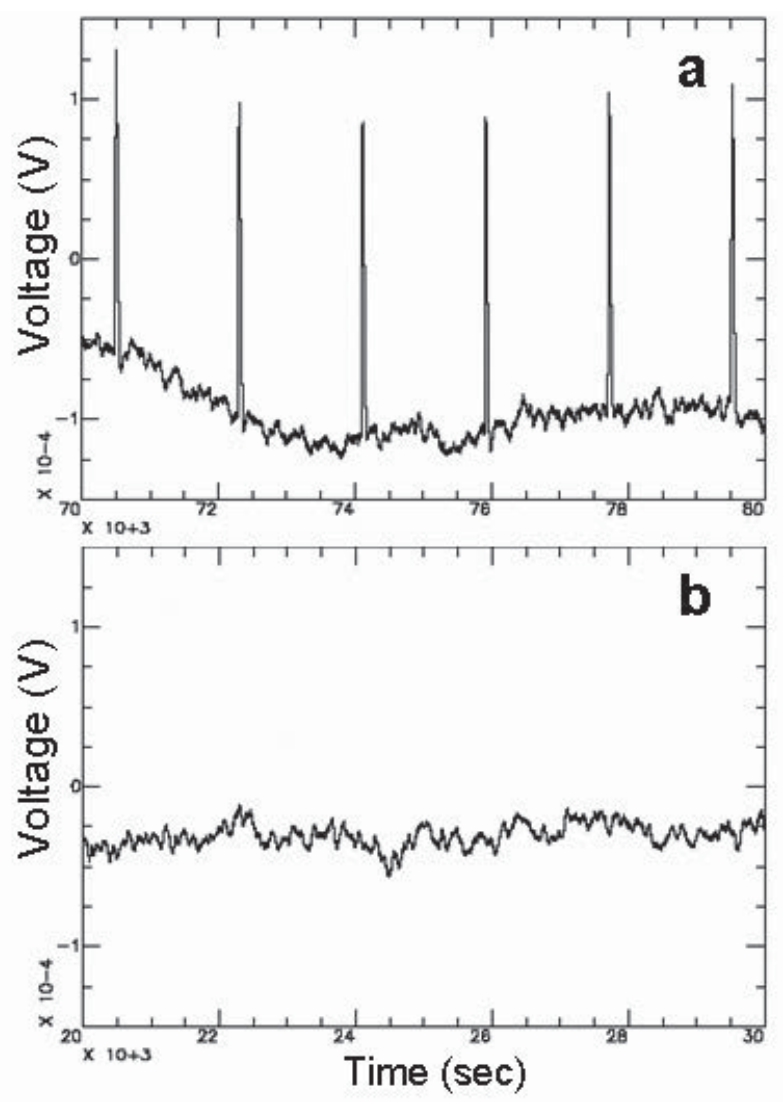

Fig.3. Removal of spike-like noises originating in the power controller (Yuki and Ishihara, 2007). (a) The wave of STS-1 Up-Down seismometer sampled at $20 \mathrm{~Hz}$ and filtered with band-pass filter between 100-10,000 sec. with the ground line between frame GNG (FG) and GND terminals. (b) The wave of STS-1 Up-Down seismometer at BAG sampled at $20 \mathrm{~Hz}$ and filtered between 100-10,000 sec. without the ground line between FG and GND terminals. Division on the horizontal axis is $500 \mathrm{sec}$. after replacement, we calibrated the seismometer to measure relative frequency response by injecting sine currents of various frequencies in the calibration coil. As a result of those calibrations, the frequency response in amplitude and phase after replacement were almost consistent with theoretical responses over all frequencies, whereas those before replacement obviously deviated from theoretical responses at frequencies lower than $0.1 \mathrm{~Hz}$ (Fig. 2). In short, this result shows the damping of free oscillation becomes stronger with a poorly insulated connector, as suggested by Yamada (2001). If this deviation in seismometer's frequency response remains without improvement, it might not influence high frequency records - those used in earthquake source studies - but may influence low frequency records, those used for earth's free oscillation studies. For this result and from our experience, in order to avoid the situation that the seismometer's connector has some conductors too poorly insulated to recover by drying them, it is very important that we protect the connectors with a plastic sheet and fasten them for effective waterproofing in every maintenance work.

\subsection{Spike-like noise originating in the power controller}

At only two of the stations where STS-1 seismometers, the Q330, the $\mathrm{PB}-14$, and the power controller of switching-mode AC-DC converter named PSC150 are installed, spike-like noises were recorded on the seismograph's vertical component. In addition, when the PB14 started running, the input voltage of the Q330 and the PB14 from the PSC150 decreased suddenly for a few seconds, while that of the STS-1 seismometers did not change at all. After we encountered this trouble, we tried to simulate the noise generation and discover how to remove the noise without losing the compact size and low cost-performance of the PSC150. As a consequence of our trials, we succeeded in simulating the noise generation and found the best method that the grounding line for the PSC150's aluminum frame should be separated from that for power return. We concluded that because the grounding line for power return, which means $0 \mathrm{~V}$, has some impedance because it is connected to the grounding frame, the voltage of the power grounding line fluctuated, and then spike-like noises were generated when some rush of current flowed (Yuki and Ishihara, 2007). By separating the two grounding lines, we could actually remove the noise at one station (Fig. 3). Kinemetrics/Quanterra, the manufacturer of the Q330 and PB-14, on the other hand, reported the instance that similar spikes appeared on the 


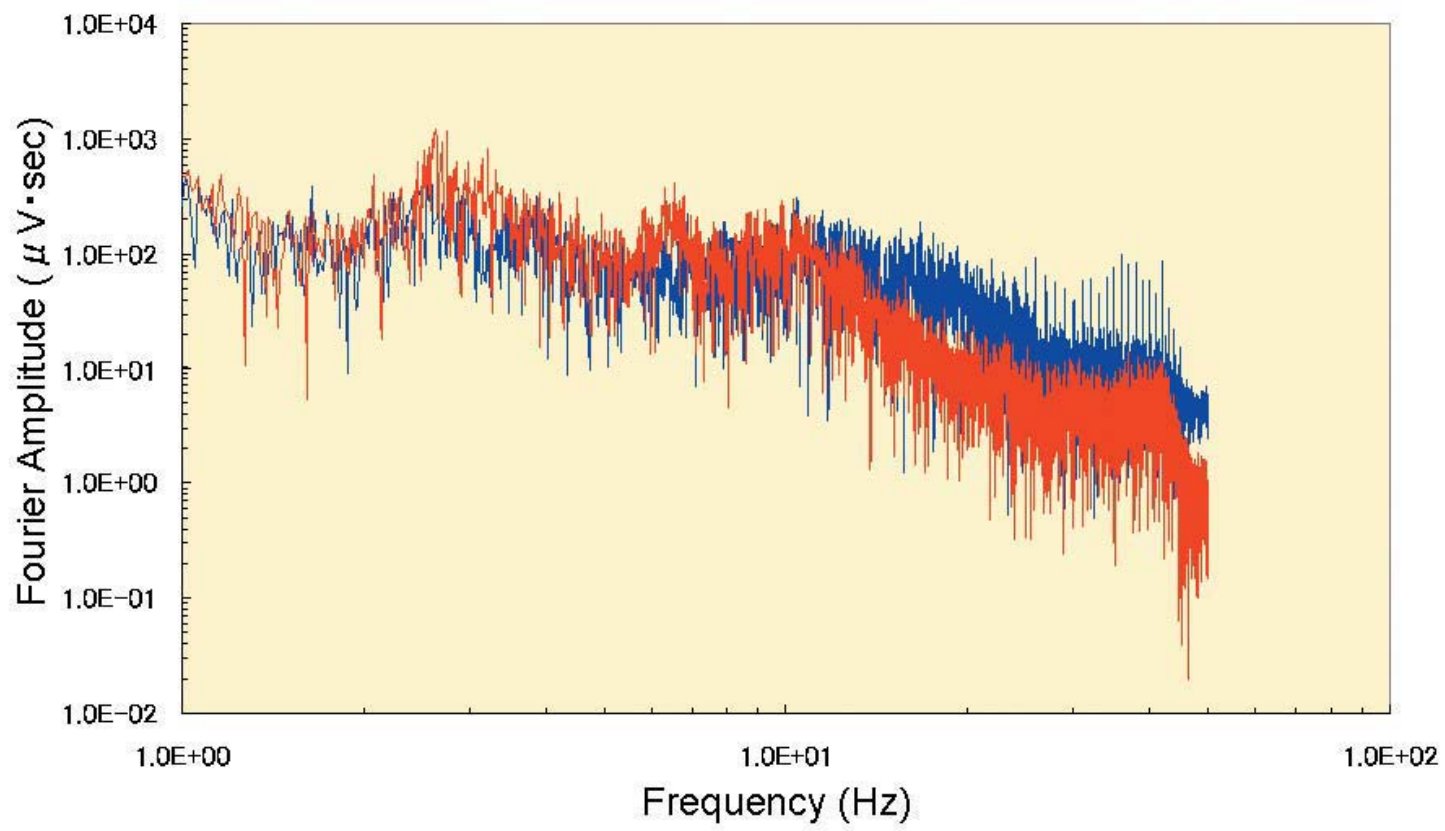

Fig.4. Spectrum of single-ended signaling and differential signaling. Blue spectrum is twice the spectrum of single-ended signaling; red spectrum is that of differential signaling. Each spectrum was estimated with FFT from $100 \mathrm{~Hz}$ data of 81.92 seconds.

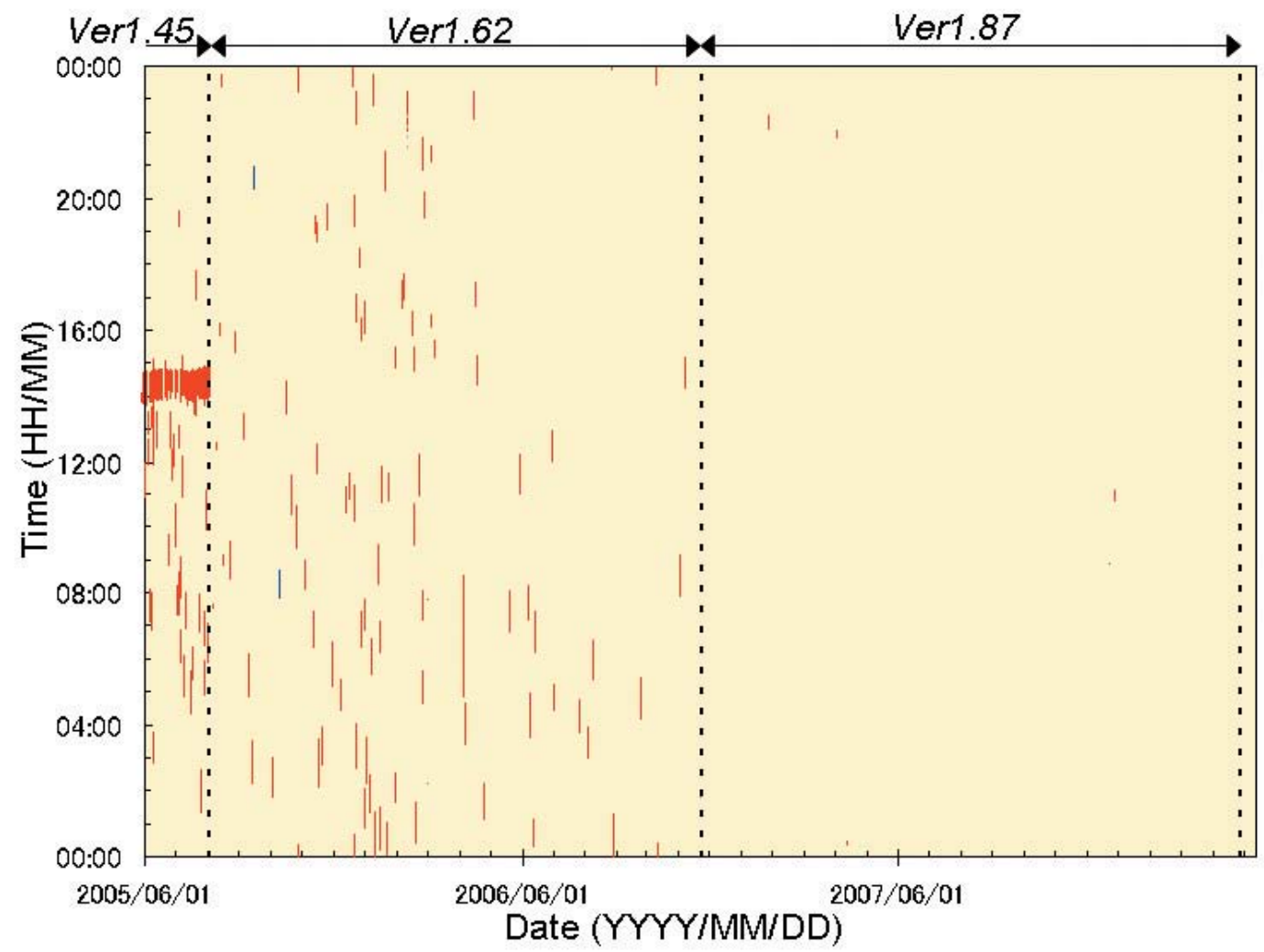

Fig.5. Data gaps in PB-14 due to automatic shutdown. The red lines indicate a data gap due to the PB-14's automatic shutdown; the blue lines indicate a data gap due to long-term power failure. 
STS-2 seismometer's output whenever the PB-14 started running (Kinemetrics and Quanterra, 2007). Furthermore, according to the same document, they recommend that Q330 and Q330HR users adopt a low-impedance power source, such as a battery operated from a constant-voltage float charger, or to equip the AC-DC converter with post-filtering and reservoir capacitors to satisfy the required peak current demands. As mentioned above, the method of grounding and stable power supply may realize sufficiently sensitive broadband observation; therefore we need to choose the best solution for retrieving electrically noiseless data within the limitations of portability and cost performance, depending on the circumstances of each station, such as the stability of the $\mathrm{AC}$ power source and grounding resistance.

\subsection{High frequency noise by one-sided connection of a differential signal pair}

The method of differential signaling which separates a signal into an inverted and a non-inverted signal and then transmits them via a pair of lines is used in many kinds of broadband seismometer, including the STS-1, STS-2, and CMG-3T. Our data logger measures the difference between the inverted and non-inverted signal, namely, the amplitude twice the original signals. A series of differential signaling systems is believed to be effective against commonmode noise interfering with signal lines with the same condition. Coincidently, at three stations we encountered the troubles that one line of differential signaling in the STS-1 seismometer was disconnected by some force. As described above, if one line of differential signaling is disconnected, which is called single-ended signaling, the absolute value of the measured signal becomes half of the complete differential signaling. Compared with the spectrum of continuous ground motion with no earthquake, for the differential signaling at a station, twice the spectrum of the single-ended signaling is obviously large in the frequency domain higher than $10 \mathrm{~Hz}$ (Fig. 4). This result implies that differential signaling can reject high frequency electrical noises invading signal lines, proving it sufficiently effective in seismic observation. In fact, it is difficult to notice a disconnected line of differential signal because the amplitude of continuous ground motion can fluctuate to twice or greater from day to day. Therefore, we should monitor data diligently in time series and spectrum, comparing old with new data and one with another component of seismometer.

\subsection{Data gaps in hard disk due to automatic shutdown}

Sometimes we had not received data from a station for a long time due to a breakdown of a networking device such as a DSL modem and router or unstable Internet condition. For this reason, the Q330's or Q330HR's dedicated hard disk of PB-14 is installed at all the stations we have been maintaining, and it retrieves data from the Q330 or Q330HR as backup data storage. However, a trouble has occurred many times at almost all stations in which the PB-14 unexpectedly stopped and then automatically started running again after a few minutes to several hours. In other words, no data was stored in the PB-14 during its shutdown. From the worst station's data gaps in PB-14 (Fig. 5), the frequency of data gaps seems to depend on the PB-14's firmware version; the PB-14 used to stop around 14:00 UT almost every day under version 1.45 ; the data gaps decreased but sometime occurred more than ten times every month under version 1.62; and there were only a few data gaps for 17 months under version 1.87. Thus it is certain that the PB-14's automatic shutdown behavior was improved with upgrades to its firmware, although we don't know why it occurs because the manufacturer did not provide convincing information. Furthermore, the frequency of the PB-14's shutdown was different at each station as well, which implies that it may depend on the Internet security of the station or the condition of the power source supplying the PB14. Going through this trouble, we learned that initially we should operate equipment with new firmware, including any brand-new equipment, for a while in our laboratory before installing it at a station so as to prevent serious trouble from occurring at the station.

\section{Conclusion and discussion}

From our experience with maintenance of broadband seismic stations and experiments in our laboratory, we have learned the characteristics of many kinds of troubles and then acquired the knowledge of how to solve some of them, such as above-mentioned troubles: (1) deviation of seismometers' frequency response with protecting the connectors against high humidity, (2) spike-like noises originating in the power controller with two solutions-disconnecting some impedance from grounding for power return and improving the power controller for supplying stable voltage to the seismograph, (3) high frequency noise from disconnection of one line in a differential signaling with a measure of checking seismograms diligently to identify it immediately, and (4) 
data gaps in the hard disk due to its automatic shutdown with the solution of updating its firmware. Based on this knowledge, some troubles can be prevented from occurring or getting worse through regular maintenance work. For example, it is desirable that we dry the equipment connectors as much as possible in our maintenance work, since the more time passes, the more moisture in the connectors is difficult to remove. In addition, some troubles can be prevented by preliminary test-running new equipment. If sufficient tests of our observation system had been conducted before their installation, step-like noise originated in our power controller or data gaps in the PB-14 wouldn't have occurred at stations. Hereafter we will create some summaries of our technical troubles, driving feedback force from our maintenances as this paper described. We hope that this paper is useful not only for present and future members of our network but also for those who are involved in geophysical observation, including seismic observation.

\section{Acknowledgements}

We have been operating geophysical stations with many scientists and technical experts from universities and JAMSTEC. Additionally, we have worked with many local institutes to maintain the stations. We thank all of them for their cooperation.

\section{References}

Ishihara, I., Y. Yuki, and Ocean Hemisphere Network Operation Group(2005), Construction of new generational OHP network (in Japanese), Abstract of Japan Earth and Planetary Science Joint Meeting 2005 (CD-ROM)

Kinemetrics Inc. and Quanterra Inc(2007), Q330 operations guide: Q330HR/Q330 operation overview of support tools and baler operation.

Mizutani, H., H. Nagao, S. Tsuboi, Y. Ishihara, N. Takeuchi, Y. Yuki, Y. Yanaka, and H. Yamaji(2007), IFREE data center: New geophysical observation data distribution system (in Japanese), Abstract of Japan Geoscience Union Meeting 2007 (CD-ROM).

Yamada, I.(2001), A problem in long-term stability of STS1 seismometer (in Japanese), Zisin, 53, 259-62.

Yuki, Y., and Y. Ishihara(2005), Methods for maintaining the performance of STS-1 seismometer. Frontier Research on Earth Evolution Vol. 2: IFREE Report 2003-2004 (CD-ROM).

Yuki, Y., and Y. Ishihara(2007), Origin of artificial noise on broadband seismograms and possible prescription to remove them, JAMSTEC Report of Research and Development 5, 21-30. 